\title{
The functional independence measure in spinal cord injured patients: comparison of questioning with observational rating
}

\author{
SS Karamehmetoğlu ${ }^{1}$, I Karacan ${ }^{1}$, N Elbaşi ${ }^{2}$, G Demirel ${ }^{1}, \mathrm{H} \mathrm{Koyuncu}^{1}$ and M Döşoğlu ${ }^{3}$ \\ ${ }^{1}$ Medical Faculty, Physical Medicine \& Rehabilitation Department, Istanbul; ${ }^{2}$ Istanbul Physical Medicine \& Istanbul \\ University, Cerrahpasa, Rehabilitation Centre, Istanbul; ${ }^{3}$ Haydarpaşa State Hospital, Istanbul, Turkey
}

Functional independence measure (FIM) is becoming widely used for all aspects of disabling diseases including spinal cord injury (SCI). It is recommended that it is rated by trained clinicians familiar with the patients. We aimed to compare the ratings of those patients who were questioned with those who were observed in a simulated environment.

Fifty patients with SCI were included in the study. They were all FIM rated by the same clinician, first by questioning and then by observation. Although observational rating took much more time than questioning there was a very strong correlation between these two different rating methods.

We can conclude that questioning SCI patients could be used as a valuable and quick way to assess the functional level of such patients. Although this does not exclude observational scoring that was generally higher and more motivational for the patient.

Keywords: functional independence measure (FIM); spinal cord injury (SCI); paraplegia; tetraplegia

\section{Introduction}

Medical rehabilitation needs accurate methods of measuring human function in a reasonable period of time. Functional independence measure (FIM) (one of the latest methods to measure disability) was developed for a standard measure of disability in six areas of functioning (18 items). ${ }^{1}$ At the beginning FIM was scored on four levels, then on seven levels. So, one can have at least 18 points and maximum 126 points. ${ }^{2}$ The reliability of this seven levels scale was tested in $1991 .^{3}$ Since, FIM was adopted as a valuable measure of activities of daily living and became widely utilized internationally. ${ }^{4-7}$

The International Medical Society of Paraplegia (IMSOP) and the American Spinal Injury Association (ASIA) accepted FIM as a standard measure of disability in those with a SCI although it was originally designed for disabled people in general. ${ }^{8}$ The scoring of FIM may be arbitrary especially for some of the items such as communication, expression, social cognition and memory. ${ }^{9}$ Furthermore, different disabilities cannot be compared with FIM and it is not a perfect tool to evaluate the quality of life. ${ }^{10}$ Despite these disadvantages, FIM is recommended for all types of disabling conditions including SCI. ${ }^{5,7,11,12}$

In this study, we aimed to compare the scoring of the FIM of those patients who were questioned and

Correspondence: SS Karamehmetoğlu, MD, Kizilelma Cad. Ömer Seyfettin sk, No 14/12 34280 Findikazade, Istanbul, Turkey those who were observed in a simulated environment by the same clinician. Similar scorings values in either method would give us a quicker and safer way of assessment of the functional level of the SCI patient.

\section{Method}

Fifty patients with SCI were included in the study. They were all FIM rated by the same clinician, first by questioning and then by observation. The conversation took place in the patient's room and questions were asked in a way that the patient could understand them completely without any confusion. More explanations were given if necessary according to the intellectual level of the patient.

The observation took place in a simulated environment. The patient was informed of the procedure and then asked to perform each item. The time spent for this was recorded.

Statistical analyses were performed using SPSS (Statistical Package for Social Science) for Windows. The Sperman correlation test was adopted and $R=0.75$ and above accepted as a positive result.

\section{Results}

The average age of the patients was $33.94(\mathrm{SD} \pm 14.59)$, 38 patients $(76 \%)$ were male and $12(21 \%)$ female. The causes of SCI were road accidents 21 cases $(42 \%)$, falls 
$15(30 \%)$, gun shot seven $(14 \%)$, others six $(12 \%)$, diving two $(4 \%)$. Eleven patients $(22 \%)$ were tetraplegic, $39(78 \%)$ paraplegic.

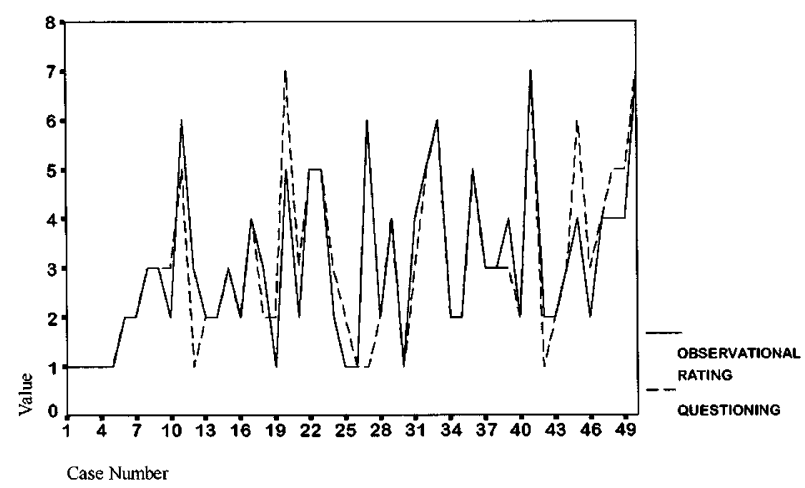

Figure 1 The comparison of the scores of the FIM for bathing $(r=0.81)$

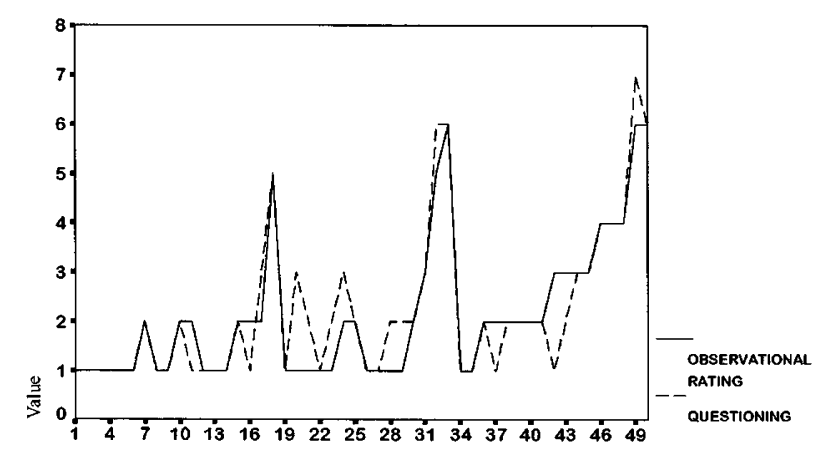

Figure 2 The comparison of the scores of the FIM for dressing the lower body $(r=0.75)$



Figure 3 The comparison of the scores of the FIM for bladder management $(r=0.90)$
The occurrence of SCI had a peak in August (12 patients, $24 \%$ ). Nearly one out of four SCI took place in this month.

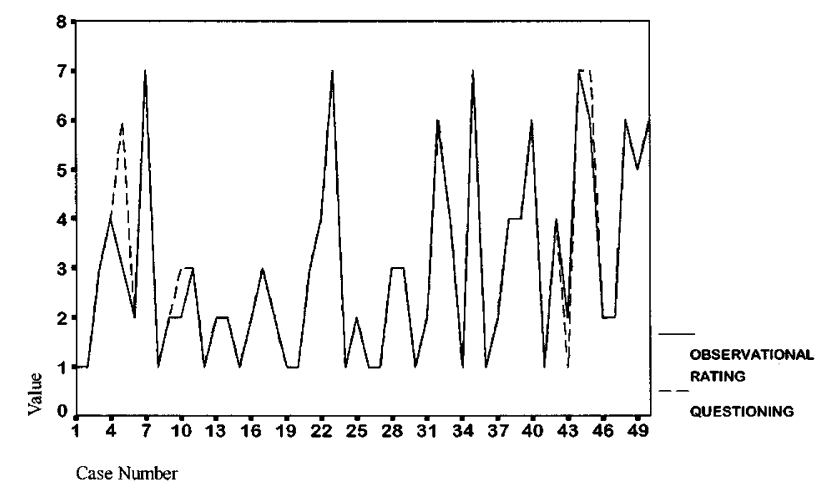

Figure 4 The comparison of the the scores of the FIM for bowel management $(r=0.97)$

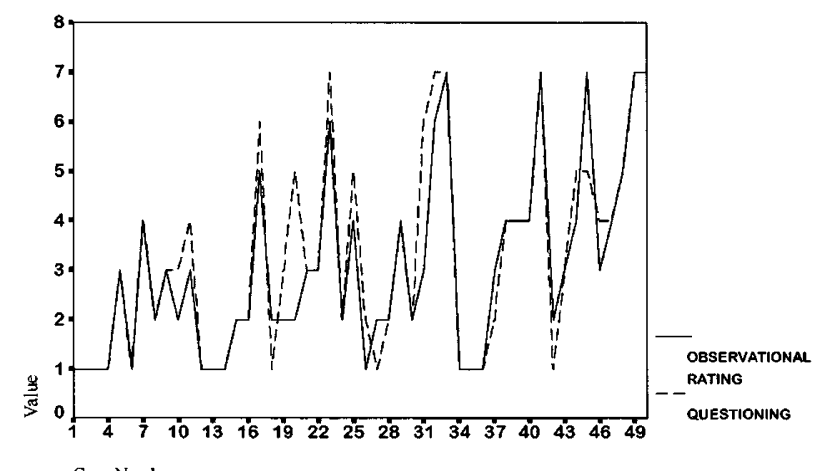

Figure 5 The comparison of the scores of the FIM for transfer to bed 0.91



Figure 6 The comparison of the scores of the FIM for walking/wheelchair $(r=0.86)$ 


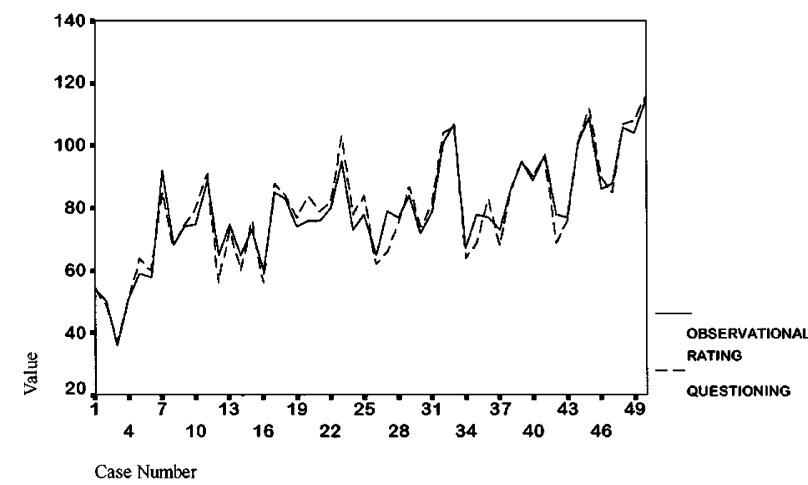

Figure 7 The comparison of the scores of the FIM for total FIM $(r=0.94)$

Twenty six patients (52\%) were primary school level educated, $17(34 \%)$ high school and three (6\%) university level educated.

There was no statistically significant difference between these two different rating methods of the FIM. Questioning and observation did not give any meaningful difference in any one of the items. They were found to be very strongly correlated $(R=0.94)$. When comparing each item the strongest were comprehension and memory $(R=1)$, the weakest was dressing of the upper body $(R=0.65)$ (Figures 1 to 7).

Observational rating took much more time (mean: $59.55 \mathrm{~min}$, SD: 25.36) than questioning (mean: $7.56 \mathrm{~min}, \mathrm{SD}: 3.63)$.

\section{Discussion}

The validity and reliability of the FIM were tested and despite some disadvantages, it was recommended because of its simplicity and feasibility in the clinical setting. ${ }^{3,6,10}$ We aimed to find out a quicker way to the use of the FIM without diminishing its value as a tool to measure disability.

The results demonstrated a very strong correlation between the FIM scores of questioning and observational ratings. The highest correlations were found in comprehension and memory. These cognitive functions do not need so much effort by the patient and are easily tested by the clinician. In Grey's study this was attributed to a ceiling effect that would require more sophisticated neurophysiological assessment. ${ }^{7}$ FIM appeared to be unable to recognize some neurocognitive and language impairments.

We had the lowest correlation in upper body dressing which demands a good deal of fine physical ability and patience from the patient and relatives. Generally, neighbouring people tend to help dress the upper body of the SCI patient. We found out that patients could do more than they thought they were able to do. Motivational approach seemed to be very important for the maximum potential of the patient.
Grey et al compared the ratings of the FIM by a clinician and the patient him/herself, and found a strong correlation between these differently rated scores. $^{7}$ It has been stated that the comparative predictive value of the questioning was consistently higher than the self-reported approach. ${ }^{6}$ In our study questioning was very strongly correlated with observational rating by a clinician. Thus questioning appeared to be more closely correlated than did self-rating. This might be due to the explanations given during questioning. Observational rating exceeded questioning and questioning exceeded self-rating on the scoring of the FIM. But, observational rating was found to be time consuming and required more manpower, compared to questioning and self-rating. Although Grey commented that self rated FIM need not to be conducted by a clinician, observational rating had the highest FIM scores by its motivational effect.

In our study, the same clinician carried out all of the examinations, thus the results can only be based on intrarater reliability. There needs to be further and larger studies to find out whether similar results would be obtained by an interrater reliability study.

We suggest that the observational rating be used for the first scoring after the acute phase of SCI and just before discharge, and questioning between these two scorings to have quick access to evaluate the course of rehabilitation in the follow-up period.

\section{Conclusion}

If one physician did the testing: Questioning the SCI patient to score the FIM appeared to be a valuable and quick way to assess the functional level of the patient; Observational rating of the FIM had generally higher scores and motivational effect for the SCI patient; Questioning might be used instead of observational rating where there is a lack of time and manpower in the rehabilitation setting.

\section{References}

1 Wade DT. Measurement in neurological rehabilitation. Oxford University Press, 1992; pp. 218-223.

2 American Spinal Injury Association (ASIA). Standard for neurological and functional classification of spinal cord injury. Revised, 1992.

3 Hamilton BB, Laughlin JA, Granger CV, Kayton RM. Interrater agreement of the seven level functional independence measure. Arch Phys Med Rehabil 1991; 72: 790.

4 Sonoda S, Tsubahara A. Functional independence measure in Japan: communication scores or social cognition and self-care activities. 1991 Academy/Congress Abstracts. Arch Phys Med Rehabil 1991; 72: 791.

5 Daverat $\mathrm{P}$ et al. The long term outcome in 149 patients with spinal cord injury. Paraplegia 1995; 33: 665-668.

6 Bunch WH, Dvonch VM. The 'Value' of Functional Independence Measure Scores. Am J Phys Med Rehabil 1994; 73 (1): 40 57.

7 Grey BA, Kennedy P. The functional independence measure: a comparative study of clinician and self ratings. Paraplegia 1993; 31: $457-461$. 
8 Hamilton BB, Fuhrer MJ (eds). In: Rehabilitation outcomes: analysis and measurement. Baltimore Brooks, 1987; pp. $137-$ 147.

9 Davidoff GN, Roth EJ, Haughton JS, Ardner MS. Cognitive dysfunction in spinal cord injury patients: sensitivity of the functional independence measure subscales vs neuropsychologic assessment. Arch Phys Med Rehabil 1990; 71: 326-329.

10 Fuhrer MJ et al. Relationship of life satistifaction to impairment, disability and handicap among persons with spinal cord injury living in the community. Arch Phys Med Rehabil 1992; 73: 552557.
11 Muecke L et al. Functional screening of lower-limb amputees: a role in predicting rehabilitation outcome? Arch Phys Med Rehabil 1992; 73: $851-858$.

12 Granger CV et al. Functional assessment scales: a study of persons with multiple sclerosis. Arch Phys Med Rehabil 1990; 71: $870-875$ 